Instituto Internacional de Investigación y Desarrollo Tecnológico Educativo INDTEC, C.A.

DOI: https://doi.org/10.29394/Scientific.issn.2542-2987.2021.6.22.20.377-396

OAl-PMH: http://www.indteca.com/ojs/index.php/Revista Scientific/oai

Ensayo Original / Original Essay

\title{
La innovación curricular frente a las realidades del docente
}

\author{
Autor: Esteban Ochoa Mena \\ Universidad Metropolitana de Educación, Ciencia y Tecnología, UMECIT \\ estebanochoa@umecit.edu.pa \\ Ciudad de Panamá, Panamá \\ https://orcid.org/0000-0001-9564-0358
}

\section{Resumen}

Una idea clara y demostrada en la historia, es que la educación como proceso transformador de las sociedades está determinada por las realidades inherentes al contexto, esta definición implica y obliga a que el currículo, como elemento fundamental y funcional de dicho proceso permanezca en constante cambio, actualización y adaptación a las comunidades educativas; en este sentido, el presente ensayo aborda desde una óptica crítico - reflexiva las tendencias curriculares contemporáneas mirando aspectos relevantes en términos de innovación, avances, perspectivas y el direccionamiento en la formación del ciudadano Colombiano; para ello, se citaron y analizaron artículos y/o publicaciones relacionadas con las nuevas tendencias curriculares, sus adaptaciones, modificaciones y reflexiones sobre su aplicabilidad en medio de la coyuntura actual del COVID-19, el impacto de las nuevas realidades afrontadas por los docentes frente al desarrollo, diseño e implementación de nuevas estrategias didácticas, pedagógicas y tecnológicas fundamentadas en la Tecnologías de la Información y la Comunicación (TIC) obligadas por la virtualidad, los cambios generacionales en la forma de obtener y producir conocimiento, los avances y perspectivas frente a la innovación curricular en Latinoamérica, y por supuesto, las expectativas tendientes en la formación de los futuros docentes. En términos generales, se trata de un escrito simple, organizado y discursivo sobre las realidades teóricas y prácticas del currículo, vistas no solo desde lo textual, sino también, desde lo orgánico, contextual y experiencial.

Palabras clave: currículo; innovación; tendencias; conocimiento; investigación.

Código de clasificación internacional: 5801.03 - Desarrollo del programa de estudios.

\section{Cómo citar este ensayo:}

Ochoa, E. (2021). La innovación curricular frente a las realidades del docente. Revista Scientific, 6(22), 377-396, e-ISSN: 2542-2987. Recuperado de: https://doi.org/10.29394/Scientific.issn.2542$\underline{\underline{2987.2021 .6 .22 .20 .377-396 ~}}$

Fecha de Recepción: 24-06-2021
Fecha de Aceptación: 08-10-2021
Fecha de Publicación: 05-11-2021 


\title{
Curricular innovation in the face of teacher realities
}

\begin{abstract}
A clear and proven idea in history is that education as a transforming process of societies is determined by the realities inherent in the context, this definition implies and forces the curriculum, as a fundamental and functional element of said process, to remain in constant change, updating and adaptation to educational communities; In this sense, this essay addresses contemporary curricular trends from a critical-reflective perspective, looking at relevant aspects in terms of innovation, advances, perspectives and the direction in the formation of the Colombian citizen; For this, articles and / or publications related to new curricular trends, their adaptations, modifications and reflections on their applicability were cited and analyzed in the midst of the current situation of COVID-19, the impact of the new realities faced by teachers in the face of to the development, design and implementation of new didactic, pedagogical and technological strategies based on Information and Communication Technologies (ICT) forced by virtuality, generational changes in the way of obtaining and producing knowledge, advances and perspectives against curricular innovation in Latin America, and of course, the expectations tending in the training of future teachers. In general terms, it is a simple, organized and discursive writing about the theoretical and practical realities of the curriculum, seen not only from the textual point of view, but also from the organic, contextual and experiential aspects.
\end{abstract}

Keywords: curriculum; innovation; trends; knowledge; investigation. International classification code: 5801.03 - Curriculum development.

\section{How to cite this essay:}

Ochoa, E. (2021). Curricular innovation in the face of teacher realities. Revista Scientific, 6(22), 377-396, e-ISSN: 2542-2987. Recovered from: https://doi.org/10.29394/Scientific.issn.2542$\underline{2987.2021 .6 .22 .20 .377-396}$

Date Received: 24-06-2021
Date Acceptance: 08-10-2021
Date Publication: 05-11-2021 


\section{Introducción}

La enseñanza como actividad natural en los seres humanos implica la transmisión de ideas, pensamientos y experiencias a través de la repetición mediante la transmisión oral o escrita de experiencias de un individuo a otro, es un proceso orgánico que incorpora elementos culturales, costumbres y hábitos como parte elemental de la actividad, Neuner (1981), citado por Navarro y Samón (2017): afirma que la "enseñanza es un sistema de acciones del maestro encaminado a organizar la actividad práctica y cognoscitiva del estudiante con el objetivo de que asimile sólidamente los contenidos [...]" (pág. 28); así las cosas, se puede considerar que la enseñanza es una práctica sin estructura implícita o explícita conducente al cambio de una aptitud, un ejercicio que se fundamenta en la asociación, la repetición y la memoria como elementos para la adquisición de una habilidad.

Contrario a lo anterior, la educación como proceso integral contempla la asociación de contenidos, estrategias, técnicas y recursos que puestos en práctica de forma ordenada conducen a la obtención no solo de aprendizajes, sino también, al desarrollo de habilidades, destrezas y conductas necesarias en la construcción del educando, del individuo o sujeto en formación.

El objetivo del presente ensayo es analizar las tendencias curriculares actuales en educación y su adaptación frente a la inclusión de las nuevas Tecnologías de la Información y la Comunicación (TIC).

\section{Desarrollo}

\subsection{Tendencias curriculares a nivel mundial y su universalización según los nuevos escenarios}

Ahora bien, a pesar de que la educación es un proceso dinámico y cambiante, para González, Pardo y Urueta-Goyena (2019): "el mundo de la educación se mueve en un nuevo tiempo, acorde con los cambios de la sociedad, que nos trae técnicas distintas de aprender y de enseñar y sobre 
Instituto Internacional de Investigación y Desarrollo Tecnológico Educativo INDTEC, C.A.

DOI: https://doi.org/10.29394/Scientific.issn.2542-2987.2021.6.22.20.377-396

OAI-PMH: http://www.indteca.com/ojs/index.php/Revista Scientific/oai

Ensayo Original / Original Essay

todo de pensar" (pág. 12); se debe percibir, como un todo producto de las reflexiones, análisis y consideraciones de la época, los problemas y situaciones contextuales.

En este sentido, se debe considerar que el proceso educativo actual no es el mismo de épocas anteriores, sino, el producto de las transformaciones subsecuentes en el tiempo, de los cambios paradigmáticos necesarios de las épocas o el resultado de las adaptaciones necesarias para lograr las metas de formación; en consecuencia, las estrategias, técnicas, materiales didácticos y pedagógicos, instrumentos, técnicas de evaluación, actividades curriculares y el currículo en general moderno son producto de las transformaciones, acciones y reflexiones que a través de los años se han venido presentando.

Una muestra de lo anterior, es lo que se viene presentando en la actualidad frente a la pandemia del COVID-19, situación que a grandes rasgos obligó de forma repentina, accidentada, poco orientada y obligada a un cambio total del currículo en las instituciones de educación en todos los niveles de formación académica, no solo en la forma de enseñanza con la inclusión de la educación remota, la virtualidad, el uso de las video llamadas a través de Google Meet, Microsoft Teams o Zoom, la creación de materiales audiovisuales o la atención constante de los chat a través de WhatsApp; sino también, un cambio en los contenidos, la estrategias de evaluación, el sistema institucional de evaluación (SIE) y hasta el proyecto educativo institucional (PEI), un cambio total del currículo; al respecto, la Comisión Económica para América Latina y el Caribe en alianza con la Organización de las Naciones Unidas para la Educación, la Ciencia y la Cultura (CEPAL-UNESCO, 2020), aseveran que:

En el ámbito educativo, gran parte de las medidas que los países de la región han adoptado ante la crisis se relacionan con la suspensión de las clases presenciales en todos los niveles, lo que ha dado origen a tres campos de acción principales: el despliegue de modalidades de aprendizaje a 
distancia, mediante la utilización de una diversidad de formatos y plataformas (con o sin uso de tecnología); el apoyo y la movilización del personal y las comunidades educativas, y la atención a la salud y el bienestar integral de las y los estudiantes (pág. 1).

Como se puede apreciar, se trata de una situación que sin lugar a dudas afecta a unos y beneficia a otros, pues, las brechas tecnológicas o de acceso a las tecnologías marca un elemento importante en el desarrollo curricular en medio de la pandemia, sin embargo, las adaptaciones emergentes diseñadas por los docentes e instituciones educativas para atender a los estudiantes han logrado disminuir la afectación alcanzando la atención de un mayor número de estudiantes, estrategias como: el desarrollo de guías moduladas, la atención por cita o agenda, la atención virtual por chat o video llamada, el desarrollo de animaciones o videos de orientación temática, el diseño de páginas web con contenido académico, el diseño de plataformas académicas con recursos de comunicación sincrónica y asincrónica, así como, el desarrollo de clases remotas, son algunos de los avances o tendencias en medio de esta pandemia.

\subsection{La adaptación del currículo frente a las tendencias actuales}

Otros elementos importantes que hay que destacar en medio de las adaptaciones que los docentes e instituciones han puesto en marcha frente a las tendencias, desafíos y retos de la actualidad, son los relacionados con las adecuaciones curriculares, pues, la situación actual ha obligado a la implementación de cambios no solo en el ¿qué?, ¿Cómo?, ¿Dónde? y ¿Por qué? del proceso educativo, se han requerido ajustes en el contenido, la finalidad de la educación, los perfiles de los educandos, el sistema de evaluación y hasta las escalas de valoración.

Así mismo, se han puesto en marcha estrategias de comunicación 
Instituto Internacional de Investigación y Desarrollo Tecnológico Educativo INDTEC, C.A.

DOI: https://doi.org/10.29394/Scientific.issn.2542-2987.2021.6.22.20.377-396

OAI-PMH: http://www.indteca.com/ojs/index.php/Revista Scientific/oai

Ensayo Original / Original Essay

sincrónica y/o asincrónica por parte de las administraciones institucionales con el fin de llevar acabo estos cambios, revisiones o reestructuraciones, estrategias como: reuniones virtuales, la actualización o modificación de documentos compartidos, los grupos homólogos, el trabajo cooperativo en línea mediante el uso de plataformas, los wikis, el intercambio mediante chat y las comunidades de aprendizaje son algunas de las estrategias diseñadas para lograr estos objetivos; al respecto de la situación actual, para Vialart (2020):

Estas disposiciones han conllevado a una transformación en los estilos y las conductas de vida en general, para intentar mantener la cotidianeidad, pero desde la distancia. Actividades habituales como el trabajo y el estudio igual han sufrido modificaciones con la misma rapidez que la aparición de la enfermedad, por tanto, se ha tenido que innovar para pasar a la virtualidad -proceso inherente a estas ocupaciones-, lo que implica un gran reto, sobre todo para aquellas personas carentes de acceso y/o conocimientos tecnológicos (pág. 3).

Ahora bien, además de los cambios en el fondo y la forma de abordar el currículo en las instituciones educativas, los avances y tendencias actuales tendientes a la universalidad de la educación han conllevado a la inclusión de elementos genéricos que rijan para todos los estamentos educativos, es decir, se han implementado elementos básicos que determinan lo mínimo que se debe saber o aprender los educandos en cada uno de los niveles de educación, pero no, como una exigencia o una estructura inequívoca que rige para todos, sino como un componente que flexible y adaptable puede aplicarse a cada uno de ellos considerando sus habilidades o capacidades, a este elemento en cuestión lo denominan en algunos escenarios: Derechos básicos de aprendizaje (DBA) entendidos según el Ministerio de Educación Nacional de Colombia (MEN, 2016) como:

[...] Los aprendizajes estructurantes para un grado y un área particular. Se entienden los aprendizajes como la conjunción 
de unos conocimientos, habilidades y actitudes que otorgan un contexto cultural e histórico a quien aprende. Son estructurantes en tanto expresan las unidades básicas y fundamentales sobre las cuales se puede edificar el desarrollo futuro del individuo (pág. 6).

Según lo anterior, no se trata solo de unificar criterios de enseñanza, sino, de homogenizar contenidos, estrategias y recursos curriculares de forma tal, que sean universales y aplicables a todas las instituciones educativas, se trata de una globalización de la educación, una internacionalización de los procesos curriculares para que sean iguales a todos.

No obstante, en otros escenarios educativos no solo se abordan los contenidos o aprendizajes mínimos como los establece el MEN en sus DBA, sino, que llegando más allá, las instituciones de educación básica y superior, han venido desarrollando e implementando estrategias curriculares adaptadas a nuevos y distintos escenarios mediante la inclusión de las tecnologías de la información y la comunicación (TIC) que han permito el diseño y desarrollo de plataformas, simuladores y ambientes virtuales de aprendizaje (AVA) que van más allá del simple currículo y permiten la deslocalización de la educación, entendiendo esto como la posibilidad de que los estudiantes accedan en tiempo real o irreal a los contenidos, practicas, materiales, recursos y herramientas del proceso educativo.

De acuerdo con Espinoza, Jaramillo, Cun y Pambi (2018): las TIC han revolucionado el proceso educativo, pues, han permitido la dinamización de los procesos de enseñanza y aprendizaje, facilitando no solo el acceso a más y mayor información, sino también, el desarrollo de habilidades y competencias cognoscitivas, y teórico-prácticas a partir del uso de simuladores, elementos multimedia y aplicaciones de realidad virtual.

Se sobrentiende entonces, que mediante la vinculación de las TIC a los procesos de enseñanza se han transformado los escenarios educativos y se 
ha dado paso a entornos virtuales que no solo se ajustan a los currículos convencionales, sino que han obligado al diseño de currículos flexibles y adaptables a estos escenarios, permitiendo a los docentes y estudiantes acceder a procesos de enseñanza en cualquier lugar del mundo.

\subsection{Cambios en la forma de producir y obtener conocimiento}

Muy bien, ya se habló suficientemente de las tendencias actuales sobre la forma como se transmite el conocimiento, es decir, del ¿cómo? y ¿Dónde?, ahora se abordarán argumentos para exponer los cambios paradigmáticos o metodológicos sobre la forma como se produce y obtiene el conocimiento, es decir, se abordará el ¿Qué?, mencionando en este aparte, las nueva practicas colaborativas, cooperativas y de hibridación para la creación y obtención del conocimiento, tomando como base la idea tendente de la dinámica del conocimiento y los cambios subsecuentes en la forma como este se produce a partir de la investigación como elemento fundamental en la búsqueda de respuestas a los problemas del entorno.

Tal como lo afirma Gutiérrez (2014): el desarrollo de actividades investigativas tutor-estudiantes conllevan de forma directa a la producción de conocimiento, pues en la búsqueda de soluciones a los problemas contextuales se organizan y elaboran estrategias que no solo fortalecen las habilidades de los estudiantes y docentes, sino que también, originan nuevos conceptos o ideas al respecto de las situaciones.

Conforme a lo anterior y la tendencias actuales, se evidencia que la investigación o los procesos investigativos son la mayor fuente de conocimiento, siempre y cuando se entienda a la investigación como un proceso organizado, sistemático y dinámico que se desarrolla en busca de soluciones a los problemas; sin embargo, la investigación por sí sola no conlleva a la obtención de conocimiento, se debe articular el proceso investigativo con elementos sociales, ya sea como punto de partida para iniciar 
la investigación o como punto final, para compartir los resultados de dicha investigación.

Se entiende entonces, que en la actualidad la obtención del conocimiento puede surgir a partir de la agrupación de procesos dinámicos, articulados y reflexivos de distintas disciplinas del saber, en donde se aprende mediante el intercambio de conceptos para la elaboración de nuevos conocimientos o mediante el desarrollo de procesos investigativos que orientados a la búsqueda de soluciones o explicaciones a los problemas sociales conllevan a la construcción de nuevos conocimientos.

No obstante, en los escenarios educativos esta ideología de búsqueda u obtención de conocimiento se ha quedado sesgada, pues, en los establecimiento educativos de educación básica y profesional no se desarrolla o implementa la investigación como una herramienta para la búsqueda de conocimiento, sino como una asignatura más del currículo, como un elemento conceptual a partir del cual se obtiene información, pero no se crea información, contrario a lo que dicen Ramírez-Elías y Arbesú-García (2019): mediante el uso de la investigación cualitativa se logra la obtención de nuevos conocimientos sobre los problemas sociales, ambientales, educativos y económicos; en este sentido, se hace necesaria una reflexión y un cambio conceptual del currículo de investigación en las instituciones de educación básica y superior, orientando las estrategias, contenidos y herramientas en investigación hacia la producción del conocimiento.

Pese a lo anterior, es importante destacar que no solo a través de la investigación se llega al conocimiento, pues, la reflexión, las experiencias y la imaginación también son fuentes de conocimiento; empero, la investigación es la única que implica la búsqueda, recolección, análisis y conclusión de un nuevo conocimiento, que como señala Hurtado (2010): es necesario reconocer que la investigación como fuente de nuevos conocimientos se debe enmarcar dentro de un proceso sistemático y organizado, con lineamientos 
preestablecidos y demostrados que le permitan al investigador, no solo contar con las estrategias de indagación, sino también, con los recursos y las técnicas para el análisis de la información.

\subsection{Avances y perspectivas del currículo en Latinoamérica}

Con relación a lo anterior y respecto a las perspectivas del currículo, países de Latinoamérica han venido implementando reflexiones y cambios curriculares tendientes a la incorporación no solo de la investigación como un componente curricular fundamental en pro de la búsqueda de soluciones a los problemas sociales, sino también, el diseño de planes de estudios con contenido curricular transversal aplicable a todas las áreas del conocimiento en donde el componente investigativo sea el elemento básico en el desarrollo del quehacer del docente y el estudiante, para que este último aprendahaciendo, contribuyendo a la búsqueda de soluciones a los problemas contextuales y aporte a la construcción de su entorno, Romero (2003): "insiste entonces en la necesidad de construir una visión de la escuela como organización que no sólo enseña sino que aprende" (pág. 6).

En este sentido, mediante la implementación del currículo transversal no solo se apunta al mejoramiento de la calidad educativa en Latinoamérica, sino también, al fortalecimiento de la educación mediante la investigación como elemento fundamental en la búsqueda de soluciones a los problemas sociales, económicos, ambientales y educativos, partiendo del conocimiento del contexto real, de la situación contextual o la realidad de los estudiantes.

Sin embargo, es necesario considerar elementos importantes al momento de hablar de investigación, pues, ésta no es solo una carta escrita o una guía preestablecida sobre ¿Cómo se debe hacer una investigación?.

Se debe principalmente, organizar un currículo especial para tal fin y vincular para este aspecto especialistas en el tema investigativo, mencionando a Villegas y González (2011): para la generación del conocimiento científico 
mediante la investigación se desarrollan procesos críticos, rigurosos, sistemáticos y lógicos, por lo que, no se trata de una estrategia de carácter intelectual, sino, instrumental, por cuanto debe ser muy bien tejida, ya que la implementación adecuada puede conllevar a la solución o no del problema u objeto de estudio; por lo tanto, además, de modificar el currículo y crear un plan de estudios, también se requiere la realización de capacitaciones o inducciones especializadas sobre la forma de hacer investigación.

Del mismo modo y tomando como punto de partida la importancia del contenido dentro del currículo, se deben diseñar planes de estudio ajustados a las realidades contextuales de los estudiantes y los docentes, se debe tener en cuenta aspectos inherentes a las instituciones, las situaciones económicas, sociales y la disponibilidad de recursos didácticos y pedagógicos, lo que implica en un sentido estricto, una participación activa de la comunidad educativa en la construcción, revisión y reestructuración del currículo.

Además, es de suma importancia, que el currículo sea abierto, flexible y adaptable al cambio generacional y las nuevas tendencias educativas, Galeano (2009): asevera que la construcción del currículo no se debe desarrollar como una obra de principio a fin, sino, como un proceso ajustable que deje espacios al mejoramiento y la adaptación a las situaciones del contexto; así mismo, el currículo y dentro de este, los planes de estudio deben ser elaborados con la participación de los docentes y los estudiantes tomando en cuenta sus intereses y preocupaciones reales frente a la vida en el aula y el mundo que los rodea.

Por lo tanto, al momento de diseñar, redactar o ajustar el currículo se hace necesario un ejercicio de concertación, socialización y flexibilización en donde todas las partes de la comunidad educativa tengan participación directa, así, se logrará un currículo realista, significativo y propio de la naturaleza institucional. 
Instituto Internacional de Investigación y Desarrollo Tecnológico Educativo INDTEC, C.A.

DOI: https://doi.org/10.29394/Scientific.issn.2542-2987.2021.6.22.20.377-396

OAI-PMH: http://www.indteca.com/ojs/index.php/Revista Scientific/oai

Ensayo Original / Original Essay

\subsection{La innovación y las TIC en la modernización del currículo}

Otro elemento importante a tener en cuenta en la elaboración del currículo y su adaptación significativa a las realidades contextuales de los estudiantes, es el fenómeno del uso de las tecnologías de la información y la comunicación (TIC) en los procesos educativos, pues, se trata de una serie de recursos, herramientas y contenidos que sin lugar a dudas transforman no solo la forma de enseñanza y/o aprendizaje, sino, todo el contenido curricular. Las TIC en la educación son sin lugar a equivocación, la tendencia más importante del siglo XX, permiten no solo el acceso a recursos digitales, aplicaciones, programas, herramientas, simuladores o contenidos digitales Offline, sino también, el acceso a un mundo de información Online a través de la navegación por internet, el ingreso a bibliotecas virtuales, el intercambio de información y el conocimiento globalizado.

No obstante, el uso de las TIC en el desarrollo del currículo no debe contemplarse como un fin, sino, como un recurso o un medio, un elemento participe del desarrollo de la clase, la estrategia o la actividad curricular, para Barreto e Iriarte (2017b): las TIC se deben usar de forma adecuada en los espacios académicos, estas deben fomentar la colaboración, la creatividad y la inclusión de los estudiantes a través del conocimiento científico y el acceso a la información.

En términos generales la elaboración del currículo, además de vincular los contenidos, las estrategias, la participación activa de la comunidad educativa y la fundamentación transversal en investigación, se debe incluir elementos tecnológicos como recursos de desarrollo teórico-práctico que además de facilitar el acceso a los contenidos, le permitan a los estudiantes aprender-haciendo y por ende alcanzar un aprendizaje más significativo. 
Instituto Internacional de Investigación y Desarrollo Tecnológico Educativo INDTEC, C.A.

DOI: https://doi.org/10.29394/Scientific.issn.2542-2987.2021.6.22.20.377-396

OAI-PMH: http://www.indteca.com/ojs/index.php/Revista Scientific/oai

Ensayo Original / Original Essay

\subsection{Implicaciones del currículo en la formación del ciudadano colombiano}

Como se ha mencionado anteriormente, el contenido curricular no solo aborda el quehacer del docente, las herramientas, recursos, estrategias, contenidos o políticas de la institución educativa, este también incluye elementos teleológicos, sociales, éticos, académicos y culturales que se pretenden alcanzar en el educando, no solo como un sujeto habido de conocimiento, sino, como un individuo social, emocional y familiar, un ser humano, una persona con sentimientos, necesidades y expectativas; en este aspecto, Daros (2012): presenta que "[...] la educación implica una forma de adquirir esa cultura que desarrolla, organiza y reorganiza (da forma a) las posibilidades de ser, en particular, las posibilidades de sentir, convivir, conocer y decidir" (pág. 21).

En el mismo sentido, la Ley General de Educación (2021a): establece que entre los fines de la educación se debe propender por el desarrollo de "[...] la personalidad sin más limitaciones que las que le imponen los derechos de los demás y el orden jurídico, dentro de un proceso de formación integral, física, psíquica, intelectual, moral, espiritual, social, afectiva, ética, cívica [...]" (art. 5); así mismo, el desarrollo de habilidades cognoscitivas que le permitan la comprensión y producción de conocimiento científico, tecnológico y experimental a través de actividades que fomenten el desarrollo del saber.

Otros elementos básicos en la formación del ciudadano colombiano contemplados en la Ley General de Educación (2021b): pero enfocadas en la construcción del educando como individuo, no como un ser social, sino como un ser intelectual, son los denominados lineamientos curriculares, que incluyen los principios básicos estructurantes del currículo a nivel institucional, elementos como: los estándares curriculares, las mallas curriculares, las competencias por área, las técnicas de enseñanza y los índices de desempeño, entre otros, enmarcan los principios y criterios básicos en la 
formación de los educandos.

No obstante, hay que reconocer que los planteamientos teóricos en la Ley General de Educación (2021b): apuntan y están diseñadas para la formación de un ciudadano Colombiano preparado, física, psíquica e intelectualmente, la realidad es muy distante de esa utopía, pues, a pesar de las adaptaciones curriculares que se hagan según las indicaciones dadas por el MEN, el acceso a los recursos, las tecnologías, los materiales, la conectividad y con ello el mundo del conocimiento se queda sesgado para la mayoría de los estudiantes en Colombia.

Conforme al Programa de las Naciones Unidas para el Desarrollo (PNUD, 2021): en las zonas rurales de Colombia no solo se evidencian preocupantes niveles socioeconómicos que limitan el acceso a servicios y bienes, sino también, unos elevados niveles de deserción escolar determinados por la ausencia de planteles educativos, docentes o medios de transporte para llegar hasta los establecimientos, en consecuencia, los estudiantes se ven amenazados por el trabajo infantil y el reclutamiento armado; de acuerdo a lo anterior, se trata de una problemática que afecta a la mayor parte de la población, a los habitantes de la zonas rurales, pues, en Colombia la mayor parte de la riqueza y las inversiones sociales en educación, salud, saneamiento básico e infraestructura se concentra en las zonas urbanas y de estas en las ciudades principales.

Así las cosas, no se trata solo de la construcción de un currículo, sino, de la implementación de dicho currículo, aunque se cuenten con un currículo bien diseñado, asequible, flexible y moderno, este se puede ver limitado por la brechas tecnologías, estructurales y profesionales que se presentan en términos de acceso a recursos tecnológicos y conectividad, la falta de infraestructura educativa y la ausencia de personal docente o administrativo, entendiendo que estos elementos impactan significativamente y de forma directa, no solo sobre la calidad de la educación de los individuos, sino 
también, sobre sus posibilidades, potencialidades y el mejoramiento de su condición social, familiar y personal.

\subsection{Tendencias curriculares sobre la formación del docente}

En todos los apartes anteriores se ha hablado de elementos propios de la estructuración del currículo, se ha hablado sobre el contenido, las reflexiones metodológicas, las estrategias novedosas, las nuevas herramientas didácticas y pedagógicas, e inclusive, sobre la incorporación de la investigación como elemento transversal, pero no se ha hablado sobre el papel del docente, sobre su formación y sobre las tendencias que se requieren en las próximas décadas; en este sentido, hay que resaltar que los docentes como agentes del proceso de aprendizaje deben ser estudiantes constantes y permanentes, adaptables, flexibles y sujetos listos para desaprender, dinámicos en mundo globalizado $\mathrm{y}$ participes de las comunidades de aprendizaje virtual.

Acorde con esto, Torre (2019): enuncia que la formación de los futuros profesores es un reto al que se enfrentan las universidades que forman para la educación básica y superior, pues, las tendencias requieren en los futuros docentes una actualización constante, que aun estimulada por los espacios universitarios se verá afectada por las condiciones sociales y el entorno de cada uno en el ejercicio de su labor.

De acuerdo con lo anterior, las tendencias futuristas apuntan a la formación de docentes con un amplio criterio de innovación e investigación, dinámicos y creativos, dotados de nuevas estrategias didácticas y pedagógicas fundamentadas en el uso e implementación de las TIC, docentes autodidactas, críticos del sistema, de los contenidos, los recursos y las herramientas, capaces de romper paradigmas en pro de la consecución de aprendizajes significativos en los estudiantes.

Es importante reconocer, que los planteamientos anteriores para 
algunos suenan como una utopía, son el deber ser del docente de las próximas décadas, pues, las realidades actuales evidencian cambios permanentes y constantes no solo en la forma de enseñar, cómo ha pasado con la llegada del COVID-19, sino también, en la forma de diseñar, elaborar y desarrollar los contenidos curriculares,

Prueba de ello es lo que está sucediendo en la mayoría de las instituciones de Colombia en donde los docentes con sus propios recursos, de forma recursiva, didáctica y tecnológica, ha diseñado contenidos multimedia, simulaciones, aplicaciones, video juegos, plataformas, páginas web, repositorios y ambientes virtuales de aprendizaje para enfrentar los cambios de la presencialidad a la virtualidad producto de la pandemia, Mendoza (2020): considera que no se trata de adoptar o no la tecnología, sino de verla como una solución tangible o inmediata a las problemáticas actuales en medio de la pandemia, que orientada hacia el desarrollo de las actividades y hacia la búsqueda de soluciones reales puede aportar frente a los cambios que se presentan.

En términos concluyentes, hay que reconocer que todo esto de la virtualidad y la modernización de la educación es difícil debido a las brechas tecnológicas existentes en algunos contextos institucionales en Colombia, sin embargo, se evidencia una preocupación marcada por parte de la mayoría de los docentes frente a cómo enfrentar este reto, tal vez no sea fácil, pero tampoco será imposible.

\section{Conclusión}

La realidad del docente, de su ejercicio profesional o su labor, se ve envuelta de forma constante y permanente en una serie de fenómenos sociales, políticos, tecnológicos y ambientales que obligan de una u otra forma a su reestructuración, ya sea en los contenidos curriculares, la forma de desarrollarlos o su finalidad. 
Es claro y necesario tener presente que con los cambios, los avances tecnológicos, las nuevas tendencias paradigmáticas acerca del currículo, las nuevas estrategias de enseñanza y aprendizaje, y el auge de las tecnologías de la información y la comunicación aplicadas a la educación, se deben hacer reformas estructurales no solo al fondo, sino también, a la forma como se desarrolla el proceso educativo.

Pues la educación como proceso, tal como se dijo al principio, implica la asociación de contenidos, estrategias, técnicas y recursos que puestos en práctica de forma ordenada conducen a la obtención no solo de aprendizajes, sino también, al desarrollo de habilidades, destrezas y conductas necesarias en la construcción del educando, del individuo o sujeto de formación, así las cosas, la educación no se debe contemplar como una rueda suelta, sino, como la base de un sistema estructurado basado en lineamientos gubernamentales claros que orienten e indiquen el norte del ciudadano en formación.

Además de lo anterior, se debe contemplar que para la correcta ejecución del currículo se hace necesaria la inclusión de recursos tecnológicos, la modernización de los espacios académicos, la formación y capacitación constante de los docentes y estudiantes, y por supuesto, la inclusión de la transversalización como una práctica formativa que conlleva al mejoramiento de la calidad educativa.

Finalmente, pero no menos importante, se debe tener en cuenta que para el fomento y la obtención del conocimiento, las instituciones educativas deben vincular y desarrollar dentro del currículo el componente investigativo, no solo como un elemento catedrático parte de una unidad temática, sino, como la base en la búsqueda de soluciones a los problemas contextuales, como la herramienta básica para dar respuestas a las situaciones sociales, educativas y ambientales de los entornos académicos; además, se debe promover en la formación de los docentes y futuros docente habilidades investigativas como fundamento esencial para la construcción del currículo de 


\section{Ensayo Original / Original Essay}

las próximas décadas.

\section{Referencias}

Barreto, C., \& Iriarte, F. (2017a,b). Las TIC en la educación superior: Experiencias de innovación. ISBN: 978-958-741-855-2. Barranquilla, Colombia: Editorial Universidad del Norte.

CEPAL-UNESCO (2020). La educación en tiempos de la pandemia de COVID-19. Chile: Comisión Económica para América Latina y el Caribe (CEPAL); Oficina Regional de Educación para América Latina y el Caribe de la Organización de las Naciones Unidas para la Educación, la Ciencia y la Cultura (OREALC/UNESCO Santiago).

Daros, W. (2012). La educación entendida como formación humana y social. Invenio, 15(28), 19-28, e-ISSN: 0329-3475. Recuperado de: https://www.redalyc.org/articulo.oa?id $=87724141003$

Espinoza, E., Jaramillo, M., Cun, J., \& Pambi, R. (2018). La implementación de las TIC en el proceso de enseñanza-aprendizaje. Revista Metropolitana de Ciencias Aplicadas, 1(3), 10-17, e-ISSN: 2631-2662. Recuperado de:

https://remca.umet.edu.ec/index.php/REMCA/article/view/46

Galeano, J. (2009). Innovar en el currículo Universitario: Una propuesta de observatorio de objetos curriculares. Uni-pluriversidad, 9(3), 314, e-ISSN: 1657-4249. Recuperado de:

https://dialnet.unirioja.es/servlet/articulo?codigo $=7895942$

González, T., Pardo, R., \& Urueta-Goyena, P. (2019). La educación experiencial como innovación educativa. México: Plaza y Valdés Editores.

Gutiérrez, N. (2014). Producción de conocimiento y formación de investigadores. Sinéctica, (43), 1-16, e-ISSN: 2007-7033. Recuperado

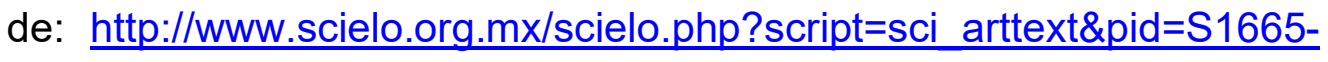


$\underline{109 \times 2014000200012}$

Hurtado, J. (2010). Metodología de la Investigación: Guía para la Comprensión Holística de la Ciencia. ISBN: 980630666X; ISBN: 9789806306660. Caracas, Venezuela: Quirón Ediciones.

Ley General de Educación (2021a,b,c). Ley 115 de 1994. Diario Oficial No. 41.214 de 8 de febrero. Colombia: EL Congreso DE Colombia.

MEN (2016). Derechos básicos de aprendizaje: Ciencia naturales. V1. ISBN: 978-958-691-923-4. Colombia: Ministerio de Educación Nacional. Navarro, D., \& Samón, M. (2017). Redefinición de los conceptos método de enseñanza y método de aprendizaje. EduSol, 17(60), 26-32, e-ISSN: 1789-8091. Cuba: Centro Universitario de Guantánamo.

PNUD (2021). Las brechas educativas en un entorno de conectividad desigual: la importancia de implementar modelos de alternancia. Colombia: Programa de las Naciones Unidas para el Desarrollo.

Ramírez-Elías, A., \& Arbesú-García, M.I. (2019). El objeto de conocimiento en la investigación cualitativa: un asunto epistemológico. Enfermería Universitaria, 6(4), 424-435, e-ISSN: 2395-8421. Recuperado de: https://doi.org/10.22201/eneo.23958421e.2019.4.735

Torre, J. (coord.). (2019). Tendencias y retos en la formación inicial de los docentes. ISBN: 978-84-8468-779-5. España: Universidad Pontificia Comillas.

Vialart, M. (2020). Estrategias didácticas para la virtualización del proceso enseñanza aprendizaje en tiempos de COVID-19. Educación Médica Superior, 34(3), 1-10, e-ISSN: 1561-2902. Cuba: Editorial Ciencias Médicas.

Villegas, M., \& González, F. (2011). La Investigación Cualitativa de la Vida Cotidiana: Medio Para la Construcción de Conocimiento Sobre lo Social a Partir de lo Individual. Psicoperspectivas, 10(2), 35-59, eISSN: 0718-6924. Chile: Pontificia Universidad Católica de Valparaíso. 


\section{Ensayo Original / Original Essay}

\section{Esteban Ochoa Mena}

e-mail: estebanochoa@umecit.edu.pa

Nacido en Quibdó, Colombia, el 21 de octubre del año

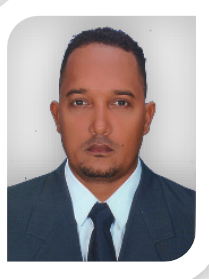
1982. Licenciado en biología y química de la Universidad Tecnológica del Chocó Diego Luis Córdoba (UTCH), Colombia; especialista en aplicación de las Tecnologías de la Información y la Comunicación (TIC) para la enseñanza; Magister en Tecnologías Digitales Aplicadas a la Educación de la Universidad de Santander (UDES), Colombia; Doctorando en Ciencias de la Educación con Énfasis en Investigación, Evaluación y Formulación de Proyectos Educativos de la Universidad Metropolitana de Educación, Ciencia y Tecnológica (UMECIT), Panamá; me desempeño como docente de ciencias naturales (biología, química y física) desde hace 10 años y estoy vinculado a la planta de docentes del departamento de Antioquia, Colombia.

El contenido de este manuscrito se difunde bajo una Licencia de Creative Commons ReconocimientoNoComercial-Compartirlgual 4.0 Internacional 